Part of Journal of Research of the National Bureau of Standards, Volume 15, September 1935

\title{
EFFECTS OF FUMIGANTS ON PAPER
}

\author{
By Charles G. Weber, Merle B. Shaw, and E. A. Back
}

\section{ABSTRACT}

The National Bureau of Standards, in cooperation with the Bureau of Entomology and Plant Quarantine of the U. S. Department of Agriculture, studied the effects of commercial fumigants on papers. This was done to find which materials could be safely used to rid valuable records of destructive insects before placing the records in the new National Archives Building. A series of papers, representative of the ordinary range used in printed and written records, was treated with the following commercial fumigants known to be effective for killing insect pests: Hydrocyanic acid gas; ethylene chloride-carbon tetrachloride; carbon disulphide; ethylene oxide-carbon dioxide; methyl formate-carbon dioxide. The papers were treated with each fumigant in a chamber used by the Bureau of Entomology and Plant Quarantine for commercial-scale fumigation work. None of the fumigants had any significant deleterious effects on any of the papers, which indicated that these chemicals can be safely used for killing insect life in records of permanent value.

\section{CONTENTS}

I. Introduction _.

II. Fumigation and testing

III. Discussion of results

IV. Summary and conclusions

\section{INTRODUCTION}

In the construction of the National Archives Building, every precaution was taken to provide the best possible protection for valuable Government records, but in order to insure the proper preservation of the materials stored in that depository, they must not be infested with injurious insect life when placed there. Boring insects, or the so-called "bookworms", can be eradicated by fumigation, and fumigating chambers have been installed in the Archives Building for use when necessary. A number of commercial preparations were known to be satisfactory from a standpoint of killing insect life; however no authentic information relative to the effects of any of the fumigants on paper was available. Obviously, the advantages of advanced practices for the preservation of records within the building would be lost if the fumigant used to prepare the records for storage there contributed to the deterioration of the paper. The effects of 5 fumigants on the chemical and physical properties of a series of papers representative of the types found in printed and written records were studied. The investigation was made at the request of the National Archives and with the assistance of personnel supplied by them. 


\section{FUMIGATION AND TESTING}

The general procedure for determining the effects of the chemicals was to fumigate specimens of the papers, and determine by chemical and physical tests the resulting changes in the paper properties. The fumigating was done by the Bureau of Entomology and Plant Quarantine in a chamber used ordinarily for the fumigation of household materials. The chamber is of metal construction, gastight, and is provided with a means of exhausting the gas and rapidly changing the air within the chamber at the completion of a fumigation. The dimensions of the chamber are 10 by $8 \mathrm{ft} 6 \mathrm{in}$.

The duration of each fumigation was $24 \mathrm{hr}$. After fumigating, all papers were allowed to season 1 week in an atmosphere of 50 percent relative humidity and $75^{\circ} \mathrm{F}$, approximately the conditions that will be maintained in the Archives Building. The papers were then conditioned in the standard atmosphere at 65 percent relative humidity, $70^{\circ} \mathrm{F}$ before testing. The fumigants used and concentrations employed were as follows: Hydrocyanic-acid gas from $1 \mathrm{lb}$ of sodium cyanide per $1,000 \mathrm{cu} \mathrm{ft}$; ethylene chloride-carbon tetrachloride, $14 \mathrm{lb}$ per $1,000 \mathrm{cu} \mathrm{ft}$; carbon disulphide, $6 \mathrm{lb}$ per 1,000 cu ft; ethylene oxide-carbon dioxide, $30 \mathrm{lb}$ per 1,000 $\mathrm{cu} \mathrm{ft}$; methyl formate-carbon dioxide, $28 \mathrm{lb}$ per $1,000 \mathrm{cu} \mathrm{ft}$. At the above concentrations, all these fumigants are 100 percent effective for killing all storage insect pests ${ }^{1}$ within $24 \mathrm{hr}$. The test specimens were suspended from cords near the ceiling of the chamber, each sheet being hung separately, with both sides exposed. The interior of the fumigation chamber and the method of exposing the paper specimens are shown in figure 1.

Eight commercial papers, 4 book papers, 3 differing as regards fiber composition and 1 with mineral coating, and 4 writing papers of different qualities were included in the study. They were tested before and after each fumigation for alpha-cellulose, copper number, acidity, and folding endurance. The alpha-cellulose content is a measure of the stable portion of the total cellulose present, while the copper number is indicative of the amount of unstable cellulose. Decrease in alpha-cellulose content, increase in copper number, and increase in acidity are all evidences of deterioration of the fibers, and the deterioration results in loss of strength, which is indicated by decrease in folding endurance. Also, both the original and the fumigated specimens were subjected to an accelerated-aging test that has been employed by the Bureau for determining the stability ${ }^{2,3}$ of papers. This was to find whether the fumigation might have a long-range effect not immediately apparent. Since approximately $4 \frac{1}{2}$ months were required for completion of the fumigation study, tests on the untreated papers were repeated to find the effects of natural aging on the papers during the period of the study. Descriptions of the papers and detailed test results are given in table 1 .

\footnotetext{
1 The following U. S. Department of Agriculture articles have been issued relating to the killing of insect pests:

E. A. Back and R. T. Cotton, Farmers Bul. 1670 and 1483

E. A. Back, R. T. Cotton, and H. D. Young, The Use of the Ethylene Oxide-Carbon Dioxide Mixture for Treating Stored Grain. Letter Circular.

E. A Back, Farmers Bul. 1353 .

W. E. Hinds, Farmers Bul. 799 .

I. E. Neifert, F. C. Cook, R. C. Roark, W. H. Tonkin, E. A. Back, and R. T. Cotton, Bul. 1313

${ }_{2}$ R. H. Rasch and G. O.'Stone, Paper Trade J. 95, T540 (July 1932).

R. H. Rasch and B. W. Scribner, BS J. Research 11, 727 (1933) RP620.
} 


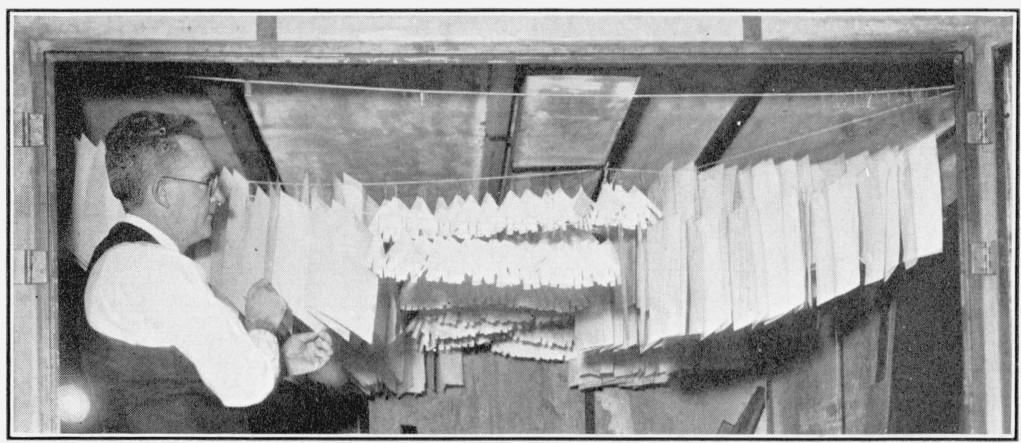

FiguRE 1.-Fumigating chamber with specimens in place for exposure. The strips are for folding endurance tests and the sheets for chemical tests. 
TABLE 1.-Effects of fumigants on properties of eight papers

[Folding-test results marked * were obtained with the MIT tester at .5-kg tension; all other tests were made by the methods of the Technical Association of the Pulp and Paper Industry]

\begin{tabular}{|c|c|c|}
\hline \multicolumn{2}{|r|}{ Papers } & \multirow[b]{2}{*}{ Fumigant used } \\
\hline$\underset{\text { ber }}{\text { Num- }}$ & Description & \\
\hline 1 & $\left\{\begin{array}{c}\text { Machine-finish; book; soda-sulphite; } \\
\text { weight } 25 \times 38: 50050 \text { pounds. }\end{array}\right.$ & $\left\{\begin{array}{l}\text { None (original paper) } \\
\text { Hydrocyanic-acid gas } \\
\text { Ethylene chloride-carbon tetrachloride } \\
\text { Carbon disulphide } \\
\text { Ethylene oxide-carbon dioxide } \\
\text { Methyl formate-carbon dioxide } \\
\text { None (original after } 41 \frac{1}{2} \text { months) }\end{array}\right.$ \\
\hline 2 & $\left\{\begin{array}{l}\text { Single-coated book; soda-sulphite; } \\
\text { weight } 25 \times 38: 50070 \text { pounds. }\end{array}\right.$ & $\left\{\begin{array}{l}\text { None (original paper) } \\
\text { Hydrocyanic-acid gas } \\
\text { Ethylene chloride-carbon tetrachloride } \\
\text { Carbon disulphide } \\
\text { Ethylene oxide-carbon dioxide. } \\
\text { Methyl formate-carbon dioxide } \\
\text { None (original after } 4 \frac{1}{2} \text { months) }\end{array}\right.$ \\
\hline 3 & $\left\{\begin{array}{l}\text { Machine-finish; book; } 50 \text {-percent rag; } \\
\text { weight } 25 \times 38: 50040 \text { pounds. }\end{array}\right.$ & $\left\{\begin{array}{l}\text { None (original paper) } \\
\text { Hydrocyanic-acid gas } \\
\text { Ethylene chloride-carbon tetrachloride } \\
\text { Carbon disulphide } \\
\text { Ethylene oxide-carbon dioxide } \\
\text { Methyl formate-carbon dioxide } \\
\text { None (original after } 4 \frac{1}{2} \text { months) }\end{array}\right.$ \\
\hline 4 & $\left\{\begin{array}{c}\text { Machine-finish; book; } 100-\text { percent rag } \\
\text { (new); weight } 25 \times 38: 50045 \text { pounds. }\end{array}\right.$ & $\left\{\begin{array}{l}\text { None (original paper) } \\
\text { Hydrocyanic-acid gas } \\
\text { Ethylene chloride-earbon tetrachloride. } \\
\text { Carbon disulphide } \\
\text { Ethylene oxide-carbon dioxide } \\
\text { Methyl formate-carbon dioxide } \\
\text { None (original after } 41 \frac{1}{2} \text { months) }\end{array}\right.$ \\
\hline
\end{tabular}

\begin{tabular}{|c|c|c|c|c|}
\hline \multirow{3}{*}{$\begin{array}{l}\text { Folding } \\
\text { endur- } \\
\text { ance (avg } \\
\text { both di- } \\
\text { rections) }\end{array}$} & \multicolumn{4}{|c|}{ Chemical tests } \\
\hline & \multicolumn{2}{|c|}{ Acidity } & \multirow[b]{2}{*}{$\begin{array}{l}\text { Alpha- } \\
\text { cellulose }\end{array}$} & \multirow[b]{2}{*}{$\begin{array}{l}\text { Coppe } \\
\text { numbe }\end{array}$} \\
\hline & $\begin{array}{c}\text { By } \\
\text { Kohler- } \\
\text { Hall } \\
\text { method }\end{array}$ & $\begin{array}{c}\mathrm{pH} \text { of } \\
\text { extract } \\
\text { (quinhy- } \\
\text { drone } \\
\text { electrode) }\end{array}$ & & \\
\hline 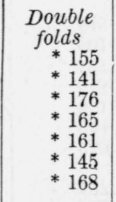 & $\begin{array}{r}\text { Percent } \\
0.054 \\
.032 \\
.032 \\
.020 \\
.008 \\
.011 \\
.020\end{array}$ & $\begin{array}{c}\mathrm{pH} \\
5.86 \\
5.80 \\
5.85 \\
5.87 \\
5.80 \\
5.80 \\
5.70\end{array}$ & $\begin{array}{r}\text { Percent } \\
80.25 \\
80.42 \\
80.02 \\
80.21 \\
80.09 \\
80.49 \\
79.75\end{array}$ & $\begin{array}{l}3 . \\
3 . \\
3 . \\
2 . \\
3 . \\
3 .\end{array}$ \\
\hline $\begin{array}{l}* 803 \\
* 849 \\
* 840 \\
* 678 \\
* 755 \\
* 831 \\
* 877\end{array}$ & $\begin{array}{l}.024 \\
.002 \\
.002 \\
.006 \\
.006 \\
.007 \\
.004\end{array}$ & $\begin{array}{l}7.60 \\
7.55 \\
7.63 \\
7.60 \\
7.68 \\
7.65 \\
7.60\end{array}$ & $\begin{array}{l}69.69 \\
72.77 \\
73.14 \\
71.11 \\
71.35 \\
71.23 \\
71.28\end{array}$ & $\begin{array}{l}3 . \\
3 . \\
3 . \\
3 . \\
3 . \\
3 . \\
3 .\end{array}$ \\
\hline $\begin{array}{l}* 161 \\
* 146 \\
* 160 \\
* 126 \\
* 162 \\
* 142 \\
* 186\end{array}$ & $\begin{array}{l}.078 \\
.080 \\
.068 \\
.077 \\
.046 \\
.063 \\
.054\end{array}$ & $\begin{array}{l}4.68 \\
4.70 \\
4.82 \\
4.65 \\
4.95 \\
4.76 \\
4.90\end{array}$ & $\begin{array}{l}85.51 \\
85.36 \\
83.18 \\
84.53 \\
84.71 \\
84.78 \\
84.58\end{array}$ & $\begin{array}{l}2 . \\
2 . \\
2 . \\
2 . \\
2 . \\
2 . \\
2 .\end{array}$ \\
\hline $\begin{array}{l}1,696 \\
1,445 \\
1,419 \\
1,617 \\
1,541 \\
1,349 \\
1,426\end{array}$ & $\begin{array}{l}.020 \\
.027 \\
.018 \\
.018 \\
.013 \\
.015 \\
.017\end{array}$ & $\begin{array}{l}5.64 \\
5.40 \\
5.45 \\
5.55 \\
5.45 \\
5.40 \\
5.35\end{array}$ & $\begin{array}{l}97.78 \\
97.50 \\
96.77 \\
97.30 \\
97.04 \\
96.91 \\
97.20\end{array}$ & \\
\hline
\end{tabular}

Heated 72 hours at $100^{\circ} \mathrm{C}$

Reten- Decrease $\mid$

\section{tion of}

endur-

ance

Increase

centage in copper

Percent 
TABLE 1.-Effects of fumigants on properties of eight papers-Continued

\begin{tabular}{|c|c|c|c|c|c|c|c|c|c|c|}
\hline \multirow[b]{3}{*}{$\begin{array}{c}\text { Num- } \\
\text { ber }\end{array}$} & \multirow{3}{*}{$\begin{array}{l}\text { Papers } \\
\text { Description }\end{array}$} & \multirow{3}{*}{ Fumigants used } & \multirow{3}{*}{$\begin{array}{c}\text { Folding } \\
\text { endur- } \\
\text { ance (avg } \\
\text { both di- } \\
\text { rections) }\end{array}$} & \multicolumn{4}{|c|}{ Chemical tests } & \multicolumn{3}{|c|}{ Heated 72 hours at $100^{\circ} \mathrm{C}$} \\
\hline & & & & \multicolumn{2}{|c|}{ Acidity } & \multirow[b]{2}{*}{$\begin{array}{l}\text { Alpha - } \\
\text { cellulose }\end{array}$} & \multirow[b]{2}{*}{$\begin{array}{l}\text { Copper } \\
\text { number }\end{array}$} & \multirow[b]{2}{*}{$\begin{array}{l}\text { Reten- } \\
\text { tion of } \\
\text { folding } \\
\text { endur- } \\
\text { ance }\end{array}$} & \multirow{2}{*}{$\begin{array}{l}\text { Decrease } \\
\text { in per- } \\
\text { centage } \\
\text { of alpha- } \\
\text { cellulose } \\
\text { content }\end{array}$} & \multirow[b]{2}{*}{$\begin{array}{l}\text { Increase } \\
\text { in copper } \\
\text { number }\end{array}$} \\
\hline & & & & $\begin{array}{c}\text { By } \\
\text { Kohler- } \\
\text { Hall } \\
\text { method }\end{array}$ & $\begin{array}{c}\mathrm{pH} \text { of } \\
\text { extract } \\
\text { (quinhy- } \\
\text { drone } \\
\text { electrode) }\end{array}$ & & & & & \\
\hline 5 & $\left\{\begin{array}{l}\text { Sulphite bond; weight } 17 \times 22: 50020 \\
\text { pounds; starch surface sizing. }\end{array}\right.$ & $\left\{\begin{array}{l}\text { None (original paper) } \\
\text { Hydrocyanic-acid gas } \\
\text { Ethylene chloride-carbon tetrachloride } \\
\text { Carbon disulphide } \\
\text { Ethylene oxide-carbon dioxide } \\
\text { Methyl formate-carbon dioxide } \\
\text { None (original after 41/2 months) }\end{array}\right.$ & $\begin{array}{r}\text { Double } \\
\text { folds } \\
167 \\
159 \\
168 \\
117 \\
128 \\
111 \\
127\end{array}$ & $\begin{array}{r}\text { Percent } \\
0.064 \\
.077 \\
.063 \\
.069 \\
.042 \\
.061 \\
.060\end{array}$ & $\begin{array}{l}\mathrm{pH} \\
4.50 \\
4.60 \\
4.56 \\
4.50 \\
4.74 \\
4.70 \\
4.55\end{array}$ & \begin{tabular}{r|} 
Percent \\
79.83 \\
80.09 \\
79.98 \\
78.77 \\
79.36 \\
78.55 \\
78.82
\end{tabular} & \begin{tabular}{l|} 
3. 02 \\
3. 04 \\
3. 34 \\
3. 31 \\
2. 99 \\
3. 28 \\
3. 19
\end{tabular} & $\begin{array}{r}\text { Percent } \\
23 \\
17 \\
21 \\
22 \\
27 \\
19 \\
16\end{array}$ & $\begin{array}{l}\text { 1. } 91 \\
\text { 3. } 64 \\
4.47 \\
\text { 1. } 94 \\
\text { 3. } 67 \\
\text { 1. } 05 \\
2.99\end{array}$ & $\begin{array}{r}0.53 \\
.46 \\
.19 \\
.43 \\
.44 \\
.32 \\
.59\end{array}$ \\
\hline 6\{ & $\left\{\begin{array}{c}25 \text {-percent-rag bond; weight } 17 \times 22: 500 \\
20 \text { pounds; starch surface sizing. }\end{array}\right.$ & $\left\{\begin{array}{l}\text { None (original paper) } \\
\text { Hydrocyanic-acid gas } \\
\text { Ethylene chloride-carbon tetrachloride } \\
\text { Carbon disulphide } \\
\text { Ethylene oxide-carbon dioxide. } \\
\text { Methyl formate-carbon dioxide } \\
\text { None (original after 41/2 months) }\end{array}\right.$ & $\begin{array}{l}650 \\
644 \\
661 \\
518 \\
567 \\
601 \\
548\end{array}$ & $\begin{array}{l}.023 \\
.037 \\
.023 \\
.027 \\
.036 \\
.026 \\
.026\end{array}$ & $\begin{array}{l}5.12 \\
5.16 \\
5.05 \\
5.06 \\
5.22 \\
5.25 \\
5.05\end{array}$ & $\begin{array}{l}\text { 89. } 02 \\
89.30 \\
88.64 \\
88.70 \\
88.71 \\
88.52 \\
88.62\end{array}$ & \begin{tabular}{l|}
2.01 \\
2.04 \\
2.11 \\
2.10 \\
2.12 \\
2.22 \\
2.18
\end{tabular} & $\begin{array}{l}62 \\
57 \\
61 \\
69 \\
62 \\
57 \\
57\end{array}$ & $\begin{array}{r}1.22 \\
2.41 \\
.91 \\
.89 \\
1.56 \\
1.08 \\
1.57\end{array}$ & $\begin{array}{l}.47 \\
.38 \\
.40 \\
.47 \\
.49 \\
.34 \\
.48\end{array}$ \\
\hline 7\{ & $\left\{\begin{array}{c}50 \text {-percent-rag bond; weight } 17 \times 22: 500 \\
20 \text { pounds; starch surface sizing. }\end{array}\right.$ & $\left\{\begin{array}{l}\text { None (original paper) } \\
\text { Hydrocyanic-acid gas } \\
\text { Ethylene chloride-carbon tetrachioride } \\
\text { Carbon disulphide } \\
\text { Ethylene oxide-carbon dioxide } \\
\text { Methyl formate-carbon dioxide } \\
\text { None (original after } 4 \frac{1}{2} \text { months) }\end{array}\right.$ & $\begin{array}{l}564 \\
496 \\
521 \\
508 \\
428 \\
447 \\
445\end{array}$ & $\begin{array}{l}.031 \\
.056 \\
.022 \\
.038 \\
.030 \\
.035 \\
.037\end{array}$ & \begin{tabular}{l|}
5.07 \\
5.05 \\
5.22 \\
5.13 \\
5.18 \\
5.30 \\
5.10
\end{tabular} & $\begin{array}{l}88.86 \\
88.94 \\
87.67 \\
88.13 \\
87.73 \\
87.80 \\
88.34\end{array}$ & \begin{tabular}{l|}
1.38 \\
1.39 \\
1.42 \\
1.38 \\
1.32 \\
1.40 \\
1.44
\end{tabular} & $\begin{array}{l}68 \\
64 \\
60 \\
67 \\
65 \\
62 \\
69\end{array}$ & \begin{tabular}{l|}
1.42 \\
2.58 \\
1.27 \\
1.74 \\
1.26 \\
.81 \\
1.04
\end{tabular} & $\begin{array}{l}.46 \\
.40 \\
.42 \\
.37 \\
.33 \\
.50 \\
.43\end{array}$ \\
\hline 8 & $\left\{\begin{array}{c}100 \text {-percent-rag bond; weight } 17 \times 22 \text { : } \\
50020 \text { pounds: glue surface sizing. }\end{array}\right.$ & $\left\{\begin{array}{l}\text { None (original paper) } \\
\text { Hydrocyanic-acid gas } \\
\text { Ethylene chloride-carbon tetrachloride } \\
\text { Carbon disulphide } \\
\text { Ethylene oxide-carbon dioxide } \\
\text { Methyl formate-carbon dioxide } \\
\text { None (original after } 41 \frac{1}{2} \text { months) }\end{array}\right.$ & $\begin{array}{l}3,309 \\
3,008 \\
2,968 \\
2,829 \\
2,822 \\
2,612 \\
2,706\end{array}$ & $\begin{array}{l}.031 \\
.047 \\
.036 \\
.032 \\
.022 \\
.033 \\
.029\end{array}$ & \begin{tabular}{l|}
5.62 \\
5.30 \\
5.46 \\
5.47 \\
5.68 \\
5.55 \\
5.50
\end{tabular} & $\begin{array}{l}97.82 \\
98.00 \\
97.16 \\
97.34 \\
97.18 \\
97.45 \\
97.70\end{array}$ & $\begin{array}{l}.98 \\
.70 \\
.84 \\
.76 \\
.86 \\
.86 \\
.94\end{array}$ & $\begin{array}{l}72 \\
71 \\
77 \\
73 \\
78 \\
75 \\
68\end{array}$ & $\begin{array}{l}.94 \\
.80 \\
.30 \\
.59 \\
.55 \\
.42 \\
.61\end{array}$ & $\begin{array}{l}.14 \\
.54 \\
.25 \\
.30 \\
.10 \\
.16 \\
.21\end{array}$ \\
\hline
\end{tabular}




\section{DISCUSSION OF RESULTS}

The results given in table 1 show the strength, chemical properties, and stability of each paper before fumigating; after fumigating with each gas; and after $4 \frac{1}{2}$ months of natural aging without fumigating. Decreased alpha-cellulose content, increased copper number, increased acidity, or loss of folding endurance after fumigation, would be indicative of harmful effects. However, the properties are related and a deteriorative effect serious enough to change one property would be expected to change one or more of the others correspondingly. Hence, changes in single properties could not be considered conclusive unless excessive.

None of the fumigants had any marked deteriorative effects on the papers. Papers 2 and 3 apparently showed some loss of folding endurance from carbon disulphide; however, in neither instance was the loss of strength accompanied by corresponding changes in the chemical properties, or loss of stability as indicated by the heat test. Some irregularity in the values for alpha-cellulose and copper number for papers 2,3 , and 5 may result from effects of the fumigants; however, no definite trend to indicate deleterious action of any fumigant is in evidence. In most instances, tests made before and after fumigating checked as well as the tests on the untreated papers before and after $4 \frac{1}{2}$ months of natural aging. From a consideration of these data, it appears that none of the 5 fumigants used had any significant effects on the properties or stability of any of the papers.

\section{SUMMARY AND CONCLUSIONS}

Normal fumigation with hydrocyanic acid gas, ethylene chloridecarbon tetrachloride, carbon disulphide, ethylene oxide-carbon dioxide, or methyl formate-carbon dioxide had no significant effects on representative book and writing papers. The results indicate that these effective fumigants, all of which are commercially available, can be safely used for ridding valuable documents and books of destructive insects, a step often necessary in preparing material for preservation in modern depositories.

The assistance of M. J. O'Leary, E. E. Creitz, J. E. Gibson, and M. Reiss in the laboratory testing is gratefully acknowledged.

Washington, July 11, 1935. 